\title{
Aportes en lifting facial masculino: diseño de las incisiones de abordaje
}

\author{
Contributions in male facelift: design of the approach incisions
}

\author{
Raúl López Bandera, Andrea Laura Pellón²
}

\section{RESUMEN}

Si bien la cirugía del envejecimiento facial es uno de los procedimientos estéticos más frecuentes en la actualidad, tanto en varones como en mujeres, el lifting facial en los pacientes de sexo masculino requiere especial atención, debido a diversos factores en los cuales difiere del mismo procedimiento en pacientes femeninas, como el uso de cabello corto, la presencia de barba y patillas, la calvicie, etc, así como el mayor celo de su intimidad y la reserva en los varones. Por lo tanto es de primordial importancia preservar la naturalidad. El propósito de este trabajo es presentar el diseño personal de las incisiones de abordaje para el lifting facial en pacientes de sexo masculino, y evaluar los resultados obtenidos.

Palabras claves: lifting masculino, incisiones, abordaje.

\begin{abstract}
Although surgery of the ageing face is one of the most frequent aesthetic procedure nowadays, both on male and female patients, face lift on the male patient requires special attention, due to different aspects that make this procedure different from female patients, such as short hair, the presence of beard and sideburns, baldness, etc., and greater privacy zeal in male patients. Thus naturalness is of paramount importance. The purpose of this article is to present our personal design in the approach of face lift on male patients, and evaluate the results achieved.
\end{abstract}

Key words: male face lift, incisions, approach

REVISTA ARGENTINA DE CIRUGÍA PLÁSTICA 2020;26(1):17-22. HTTPS://DOI.ORG/10.32825/RACP/202001/0017-0022

\section{INTRODUCCIÓN}

La cirugía del envejecimiento facial es uno de los procedimientos estéticos más frecuentes en la actualidad, y si bien en sus comienzos era casi exclusivamente requerida en pacientes de sexo femenino, hoy en día es demandada por pacientes de ambos sexos.

La historia de estas cirugías ha sido revisada por autores como Rogers, González Ulloa, Rees, Wood-Smith y Barton. Todavía existen dudas sobre quién fue el primero en practicar un lifting, ya que los cirujanos que las realizaban eran muy reticentes a exponer las técnicas que empleaban, en parte debido a que era una cirugía considerada "de la vanidad", y en parte por celo profesional.

Los primeros datos registrados datan de comienzos del siglo XX, con las cirugías descriptas por Joseph, Hollander, Lexer y Noèl, y consistían esencialmente en la extirpación de fragmentos elípticos de piel facial en los pliegues naturales, en la línea de im-

1. Médico Cirujano Plástico, miembro titular de SCPNEA y SACPER. lopezflag@hotmail.com

2. Médica Cirujana Plástica, miembro titular de SCPBA. andreapellon@yahoo.com.ar

$\triangle$ Correspondencia: lopezflag@hotmail.com; andreapellon@ yahoo.com.ar

Los autores no declaran conflictos de intereses

Recibido: 05/08/2019 / Aceptado: 20/12/2019 plantación pilosa, la frente y las regiones auricular y temporal, con la finalidad de estirar la piel y mitigar la caída de las mejillas y la presencia de arrugas. Uno de los primeros tratados sobre el tema fue la obra de la cirujana francesa Mme. S. Noèl, quien describió con detalles las técnicas empleadas.

Bettman, en 1920, fue el primero en describir una incisión continua temporal, preauricular, posauricular y mastoidea, que es la empleada en la actualidad, con algunas modificaciones ${ }^{1}$.

El motivo de esta publicación es mostrar una contribución personal en el diseño de las incisiones de abordaje en la técnica quirúrgica del lifting facial en pacientes masculinos, y evaluar los resultados obtenidos.

\section{MATERIALES Y MÉTODOS}

Se realizó un estudio retrospectivo sobre $135 \mathrm{pa}-$ cientes de sexo masculino, con edades comprendidas entre los 40 y los 80 años y un promedio de 63 años, los cuales presentaban signos de envejecimiento facial, todos ellos operados entre los años 1994 y 2019, en la práctica privada, con incisiones de diseño propio.

Los resultados fueron evaluados acerca del aspecto estético final por tres colegas especialistas en forma independiente, con una escala de 1 a 5 , en la que 1 es malo, 2 regular, 3 bueno, 4 muy bueno y 5 excelente. La misma evaluación se solicitó a los pacientes. 


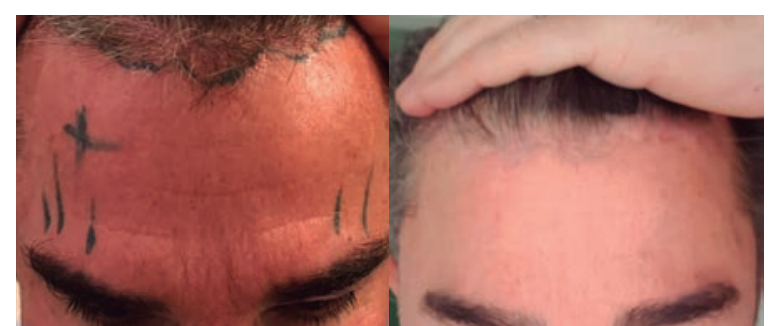

Figura 1. Incisión frontal: marcación (izq) y posoperatorio (der).

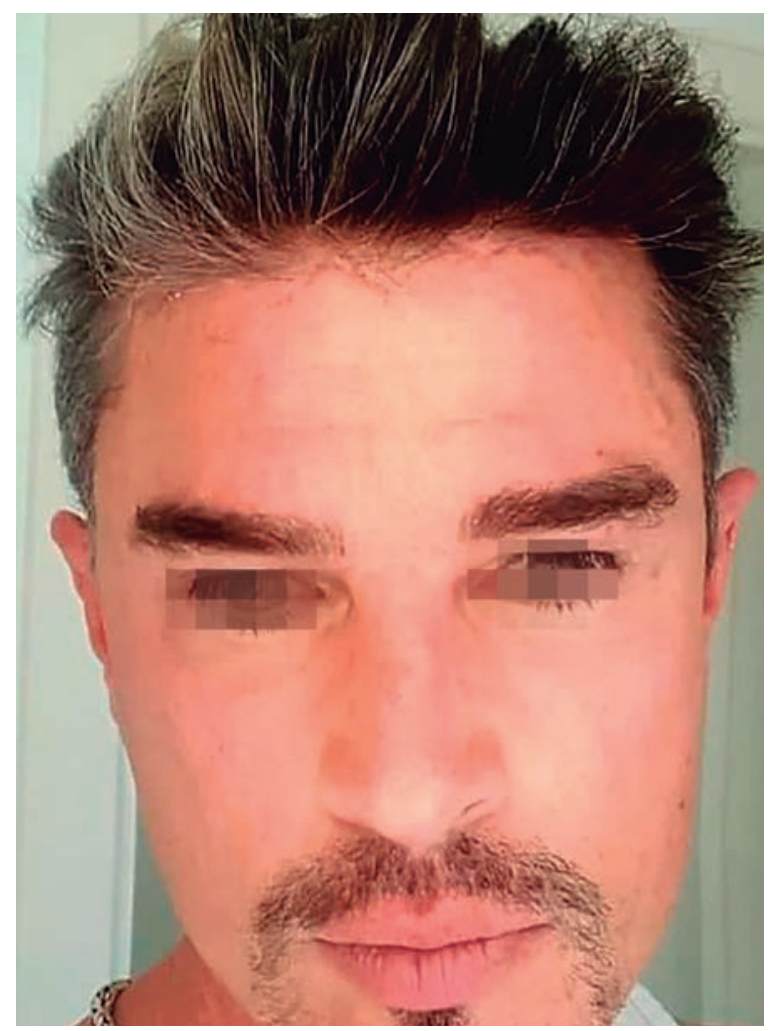

Figura 3. Incisión frontal: posoperatorio alejado.

\section{TÉCNICA QUIRÚRGICA}

Existen varios factores a tener en cuenta en el lifting facial en el hombre que lo diferencian del mismo procedimiento en pacientes de sexo femenino. En primer lugar mencionaremos el uso de cabello más corto en los varones que en las mujeres, además de la presencia de patillas y la eventual calvicie de diferentes grados. A esto se agrega la escasa posibilidad de disimular dichas cicatrices con maquillaje, lo cual obliga a ser muy precisos en la localización y calidad de dichas cicatrices. También hay que tener en cuenta la presencia de barba, que requiere especial cuidado en las zonas del trago y preauricular. Observamos también que en general los hombres suelen ser mucho más reservados y celosos de su intimidad que las mujeres en lo que respecta a la cirugía y que son menos proclives a hacer comentarios sobre ella, por lo cual la naturalidad es de primordial importancia. Además, los varones suelen ser expuestos a mayor escrutinio cuando se detectan alteraciones faciales ${ }^{2-5}$.

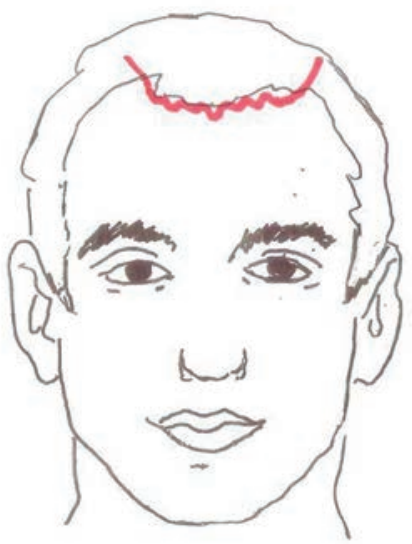

Figura 2. Esquema de incisión frontal.
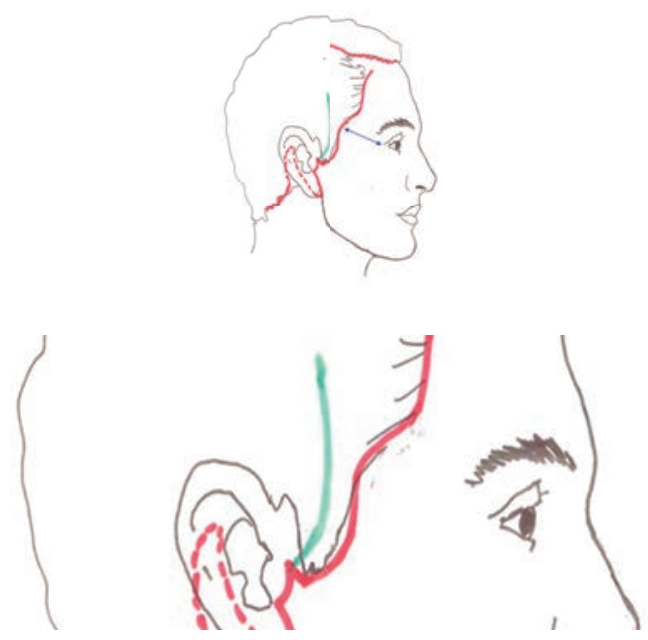

Figura 4. Incisión temporal: esquema.

Con el fin de conservar el aspecto natural del rostro masculino, diseñamos las incisiones de abordaje que se detallan a continuación

- Incisión frontal. Esta incisión tiene la finalidad de preservar las características propias de esta zona en los pacientes varones, como la frente más ancha y la menor densidad capilar, con o sin presencia de entradas; se practica una incisión que sigue en la porción central la línea de implantación pilosa normal del paciente, con un trazo de forma irregular, sin unirse con las incisiones restantes. La cicatriz resultante es menos visible, ya que no presenta un trazo lineal. Al compensar el colgajo a la altura de la cola de las cejas pueden disminuirse, y hasta eventualmente desaparecer, las entradas ${ }^{6}$ (Figuras 1 a 3 ).

- Incisión temporal. Se toma como parámetro la distancia diagonal entre el canto externo y la lí- 


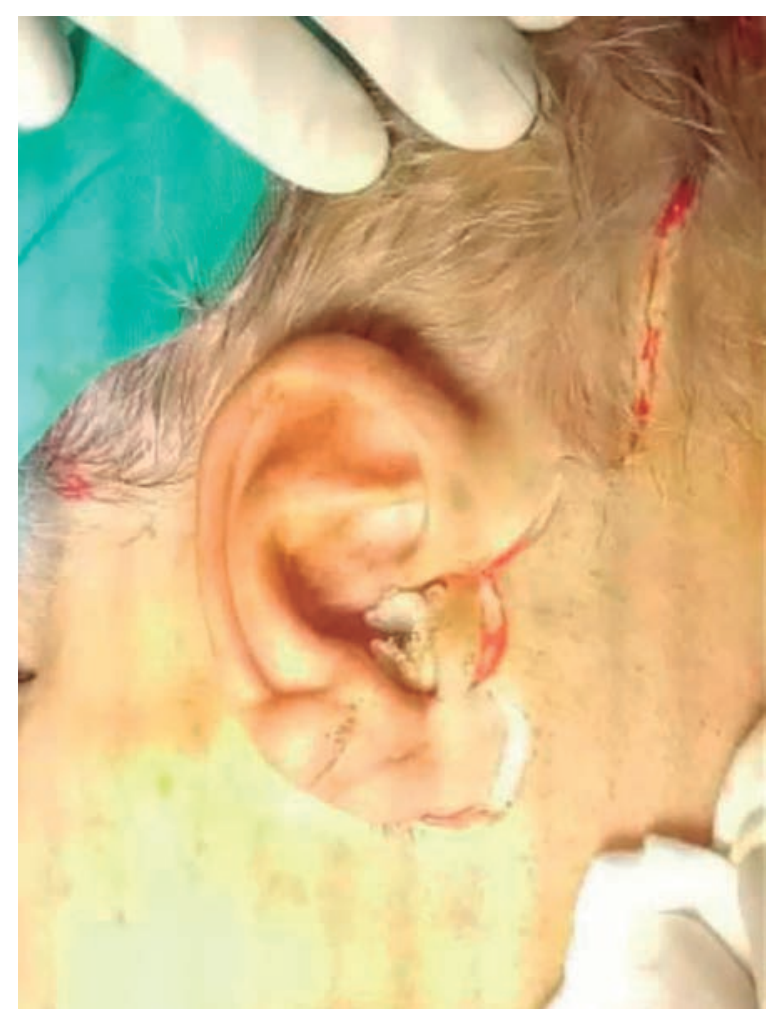

Figura 5. La incisión temporal asciende en forma de S itálica.

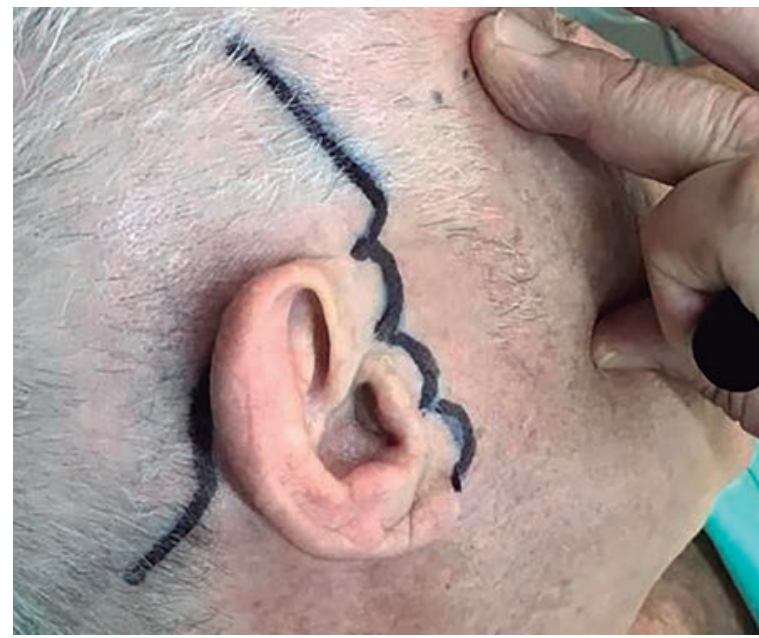

Figura 9. Marcación de la incisión preauricular.

nea de implantación pilosa para el diseño de la incisión en esta zona. Si la distancia es mayor a $4 \mathrm{~cm}$, utilizamos el abordaje precapilar a fin de evitar aumentar dicha distancia, lo cual daría un aspecto de mayor edad al paciente. Si, por el contrario, la distancia es menor a los $4 \mathrm{~cm}$, preferimos el abordaje intracapilar, con una incisión que asciende desde $3 \mathrm{~mm}$ por debajo de la patilla hacia la región temporal, con diseño en forma de $S$ itálica.

En este punto es importante evitar que la patilla ascienda por sobre la raíz del hélix, detalle que también da apariencia de mayor edad ${ }^{7}$ (Figuras 4 a 8).

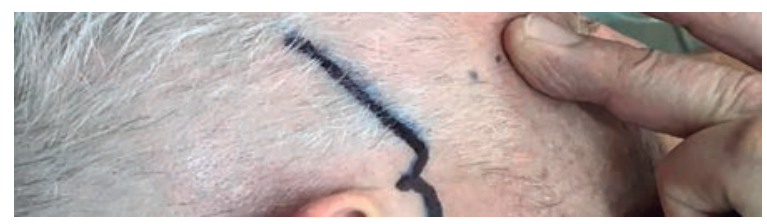

Figura 6. Marcación.

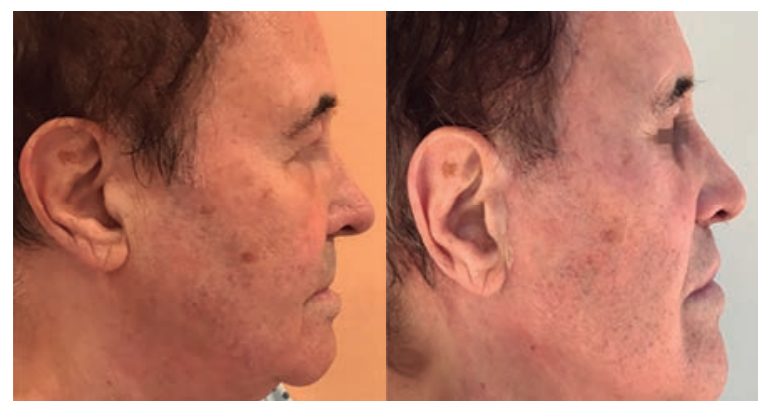

Figura 7. Incisión temporal prepilosa: preoperatorio (izquierda) y posoperatorio (derecha).

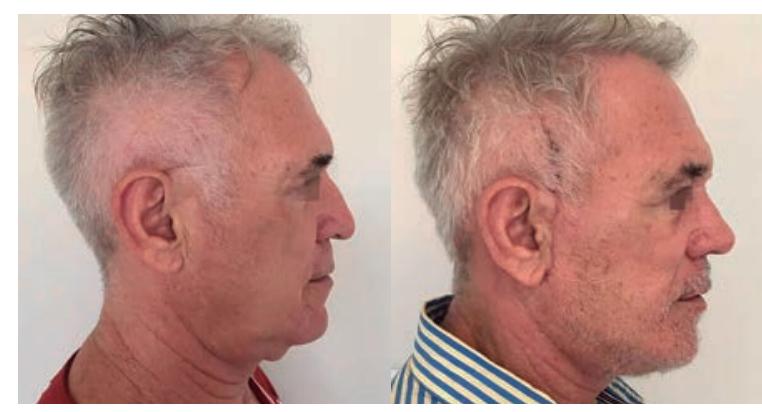

Figura 8. Incisión temporal intrapilosa: preoperatorio (izquierda) y posoperatorio (derecha).

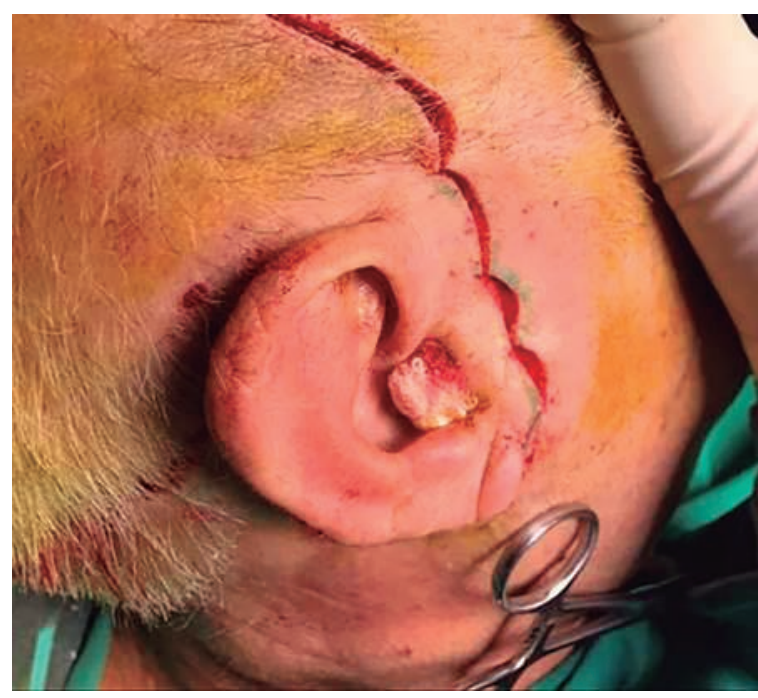

Figura 10. Incisión preauricular: intraop.

- Incisión preauricular. La localizamos pretragal en pacientes con mucha barba e intratragal en pacientes con barba más escasa, agregando en este último caso la cauterización de folículos pilosos. En ambos casos se despega el trago, para conservar la depresión pretragal. Es de vital importancia la preservación de las subunidades estéticas para lograr un resultado natural: 


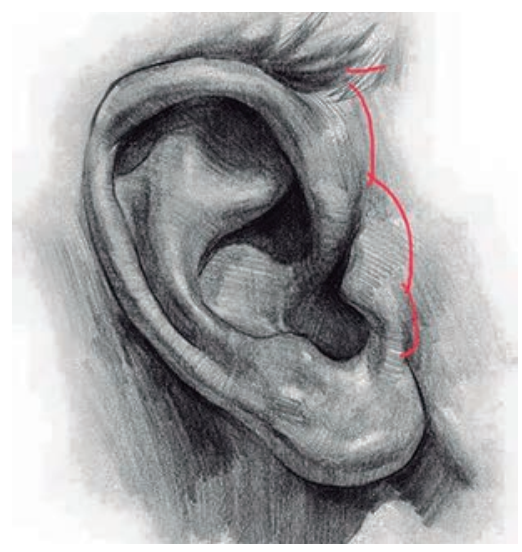

Figura 11. Abordaje pretragal.

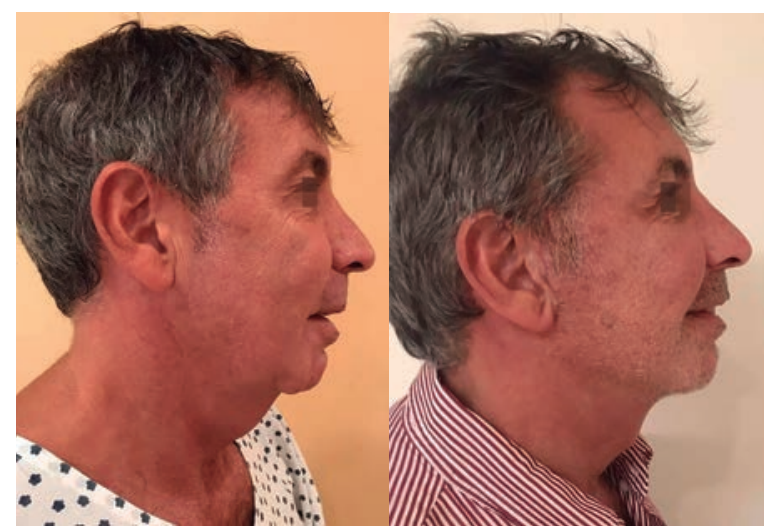

Figura 13. Abordaje pretragal: preoperatorio (izquierda) y posoperatorio (derecha).

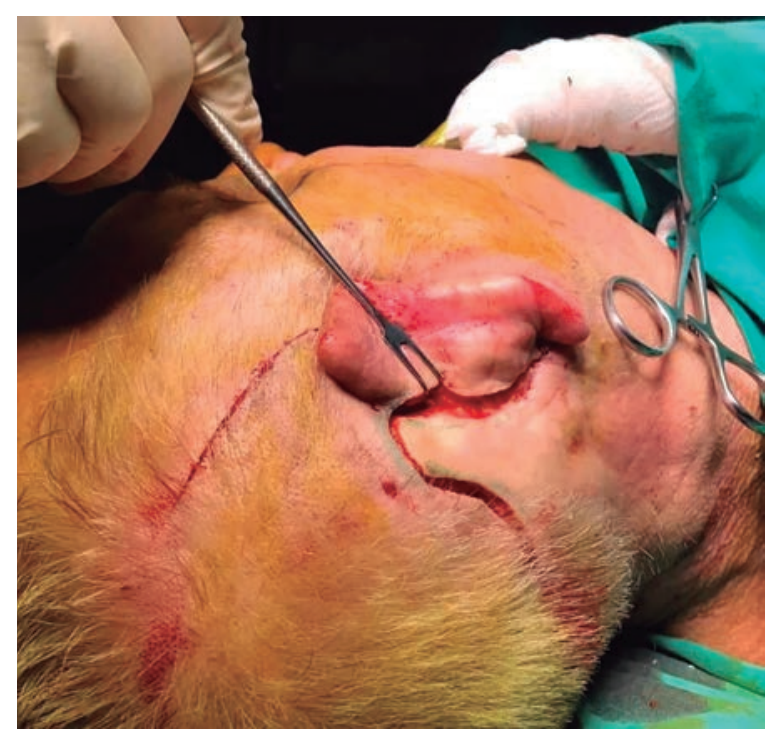

Figura 15. Incisión retroauricular.

- Ancho del hélix.

- Depresión pretragal.

- Altura del trago.

- Escotadura superior e inferior del trago.

- Unión del lóbulo auricular con la mejilla.

El diseño de la incisión preauricular debe respetar las subunidades precedentes.

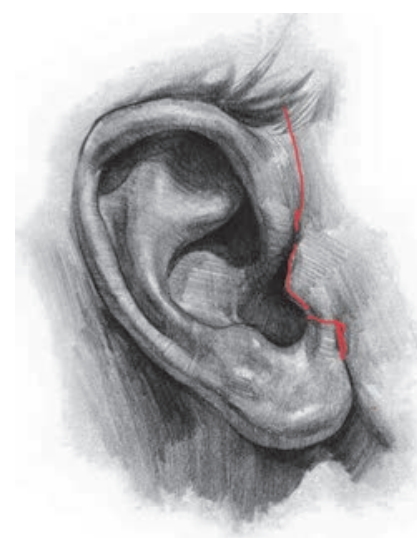

Figura 12. Abordaje intratragal

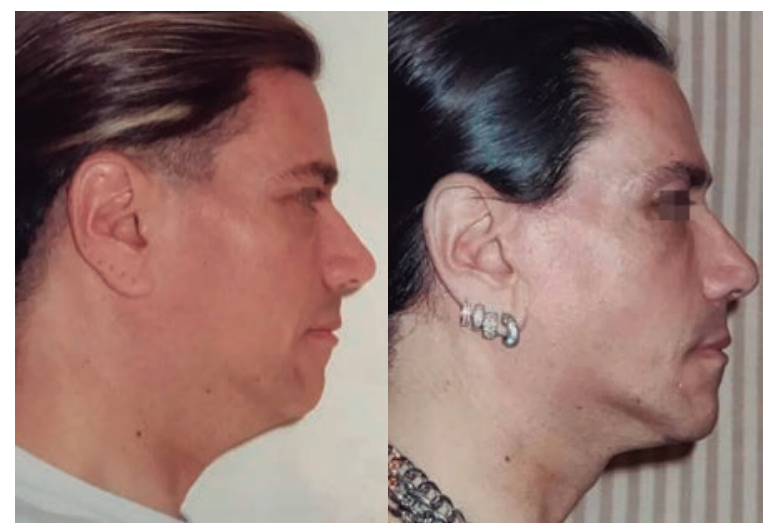

Figura 14. Abordaje intratragal: preoperatorio (izquierda) y posoperatorio (derecha).

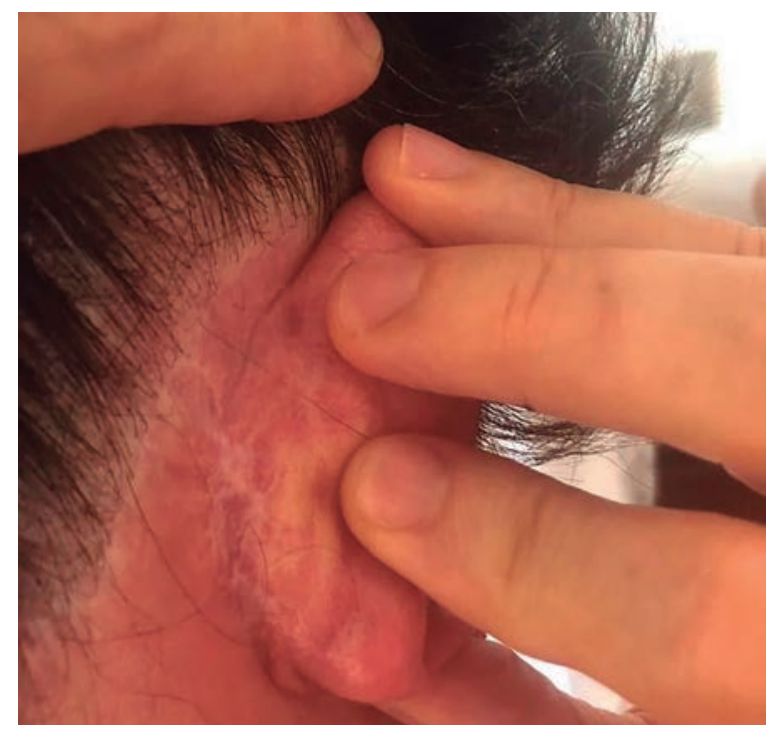

Figura 16. Incisión retroauricular: posoperatorio.

En el abordaje pretragal, el diseño de la incisión es bilobulado (Figuras 9 a 14).

- Incisión retroauricular. Preferimos ubicarla en el hueco aurículo mastoideo. Para disimular la cicatriz, se asciende por encima del nivel de la proyección del trago. Si bien de esta forma se dificulta la compensación del colgajo, el resultado estético es superior, ya 


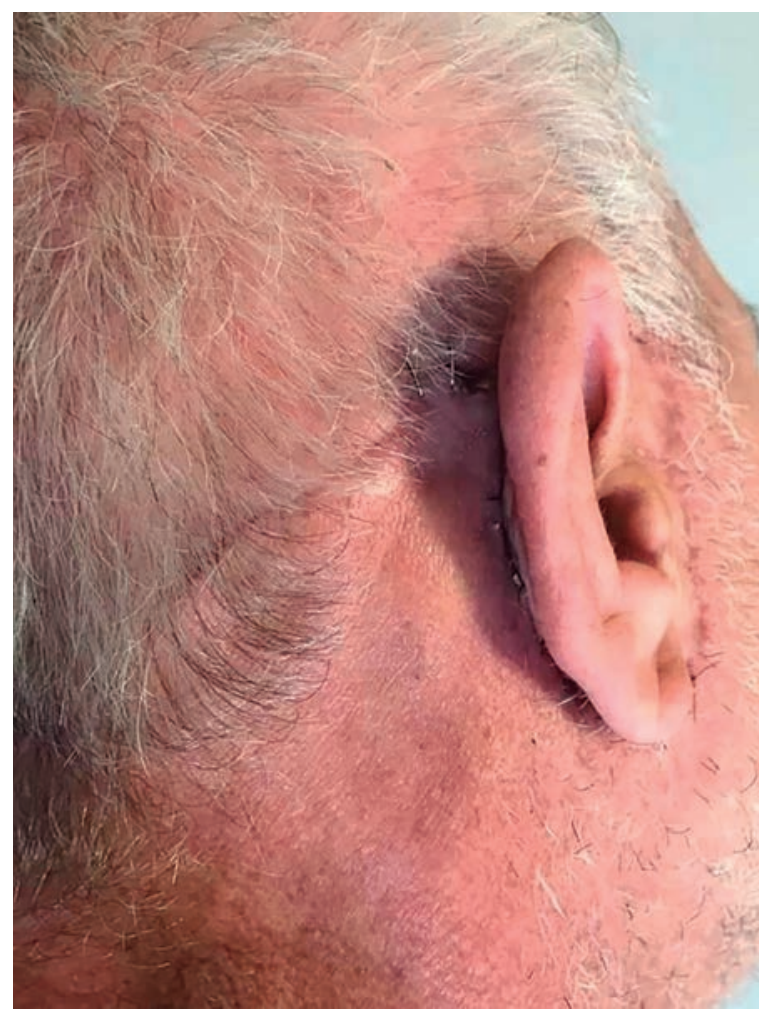

Figura 17. Incisión occipital.

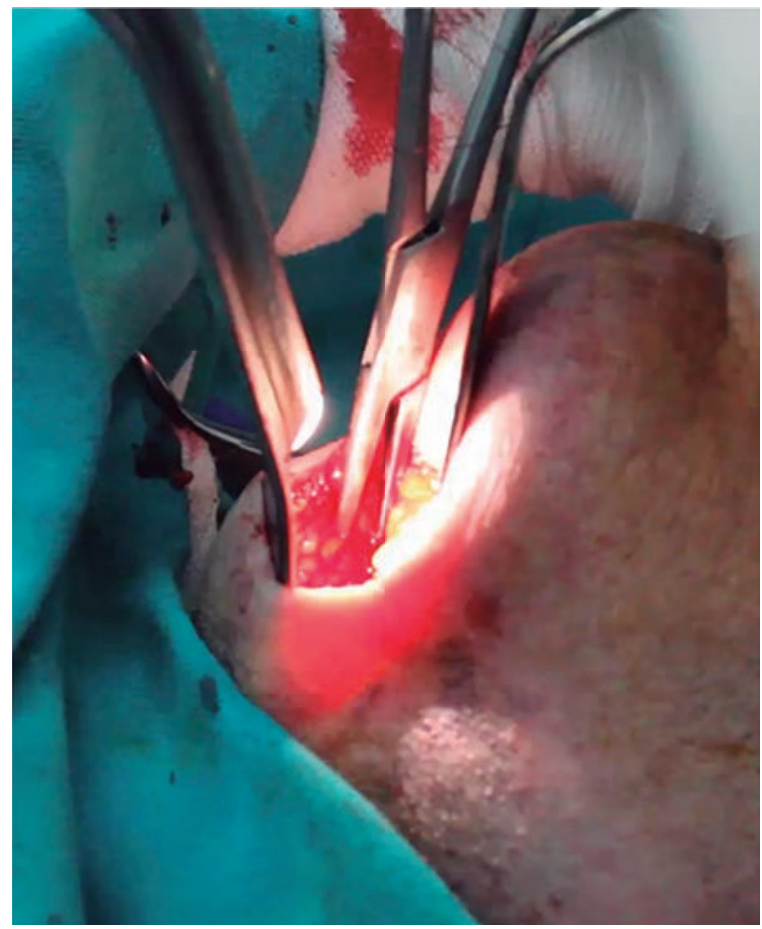

Figura 20. Incisión submentoniana: intraoperatorio.

que disminuye la percepción de la cicatriz (Figuras 15 y 16).

- Incisión occipital. En esta zona, es fundamental preservar la normalidad de la línea capilar, asegurándose que ésta no ascienda.

Si la implantación pilosa es alta, la incisión es

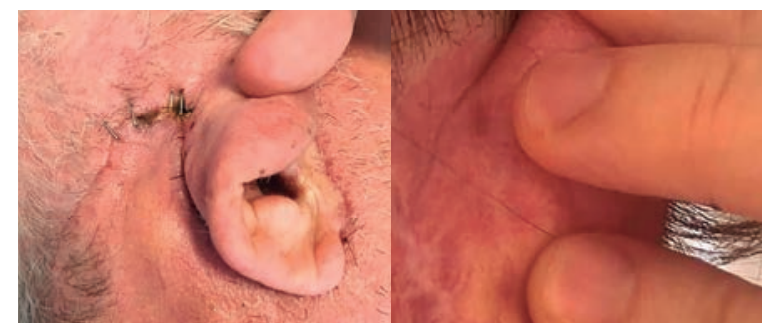

Figura 18. Incisión occipital intrapilosa (izquierda) y precapilar (derecha).

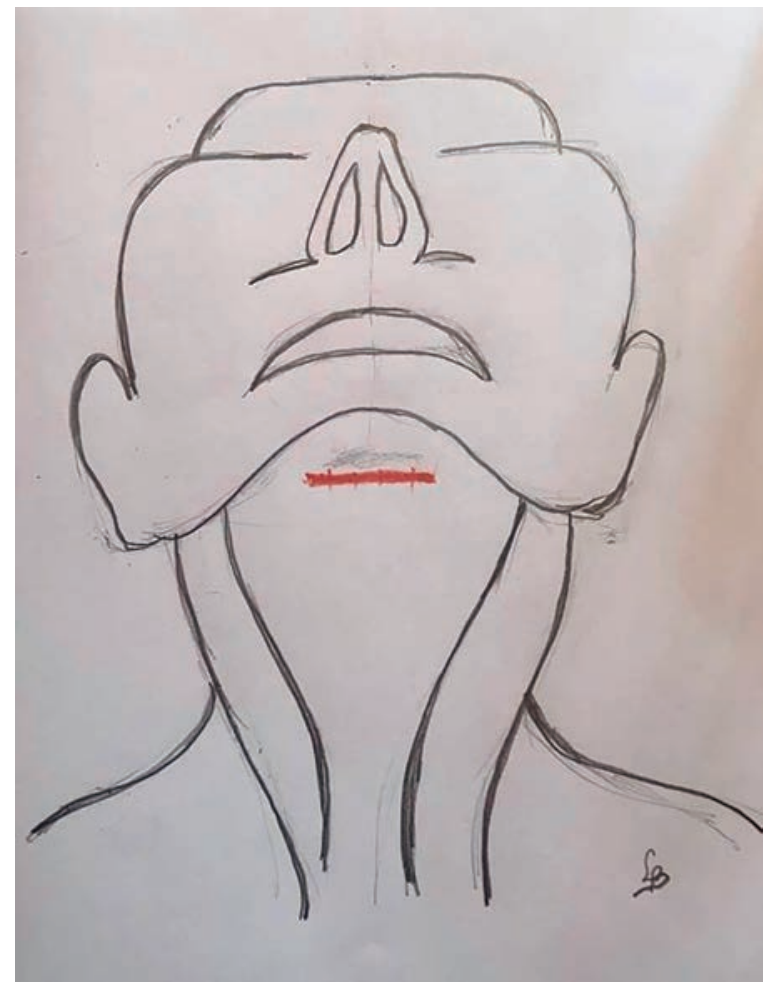

Figura 19. Incisión submentoniana: marcación.

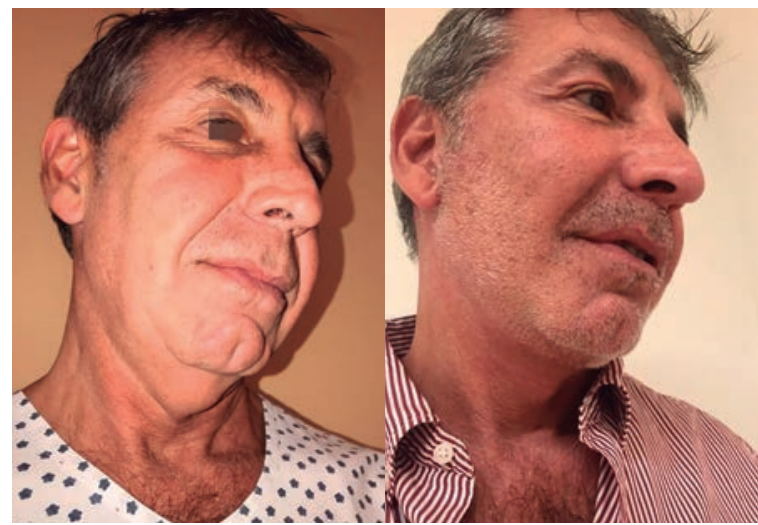

Figura 21. Incisión submentoniana: pre- (izq) y posquirúrgico (der).

precapilar. En cambio, si la implantación es normal o baja, la incisión es intrapilosa (Figuras 17 y 18).

- Incisión submentoniana. Se localiza 1 a $2 \mathrm{~cm}$ por detrás del surco, con una longitud de la misma de aproximadamente $2,5 \mathrm{~cm}$ (Figuras 19 a 21). 


\section{RESULTADOS}

Las imágenes (Figuras 1 a 21) muestran el diseño propio de las incisiones, ycorresponden a fotografías pre- $y$ posoperatorias.

Los tres profesionales consultados dieron los siguientes resultados:

- El 90\% (122 pacientes) de 3 a 5 (bueno, muy bueno y excelente).

- El 9,3\% (12 pacientes) 2 (regular).

- El 0,7\% 1 (1 paciente) malo (por cicatriz queloide).

Entre los pacientes, los resultados fueron los siguientes:
- $\quad$ El 97,7\% (131 pacientes) describieron resultados 4 y 5 (muy bueno y excelente).

- El 1,9\% (4 pacientes) 2 y 3 (regular y bueno).

- El 0,4\% (1 paciente) 1 (malo).

\section{CONCLUSIONES}

El empleo de este diseño para las incisiones de abordaje en el lifting facial en pacientes masculinos permite obtener resultados naturales y estéticamente satisfactorios, con cicatrices muy poco perceptibles, cuyo diseño está directamente relacionado en cada paciente con la implantación pilosa y la cantidad y calidad de su barba ${ }^{8}$.

\section{BIBLIOGRAFÍA:}

1. Rogers BO. Breve Historia de la Cirugía Estética. Clínicas Quirúrgicas de Norteamérica 1971;265-87.

2. Connell BF, Sundine MJ. Aesthetic Rejuvenation of the Face and Neck. Thieme Medical Publishers Inc 2016;93-101.

3. Baker TJ, Gordon TJ. Rhythidectomy in Males. Plastic Reconstructive Surgery 1969;44:219.

4. Webster RC, Fanous N, Smith RC. Male and Female Face-Lift Incisions. Arch Otolaryngology 1982;108(5):299-302

5. Little AC, Jones BC, Penton-Voak IS, Burt DM, Perret DI. Partnership Status and Temporal Context of Relationships Influence Human Female Preferences for Sexual Dimorphism in Male Face Shape. Proc Biol Sci 2002;269:1095-100.
6. Rohrich RJ, Stuzin JM, Ramanadham S. The Modern Male Rhytidectomy: Lessons Learned. Plastic and Reconstructive Surgery 2017; 139(2):295-307.

7. Guyuron B, Watkins F, Totonchi A. Modified Temporal Incisions for Facial Rhytidectomy: an 18 Year Experience. Plastic and Reconstructive Surgery. 115(2): 609-616.

8. Parsa KM, Gao W, Lally J, Davison SP, Reilly MJ. Evaluation of Personality Perception in Men Before and After Facial Cosmetic Surgery. JAMA Facial Plastic Surgery 2019; 463-480 